\title{
Interactive effects between flexible and rigid control of eating behavior on body weight: a moderated serial multiple mediation model
}

\begin{abstract}
BACKGROUND
Cognitive restraint of eating can be subdivided into rigid control and flexible control of eating behavior. Flexible control appears to be a more favorable dieting strategy as it relates to lower disinhibited eating and body mass index (BMI), while the opposite is found for rigid control. Yet, previous findings also suggest interactive effects between the two such that rigid control is particularly related to higher BMI when flexible control is low.
\end{abstract}

\section{PARTICIPANTS AND PROCEDURE}

Data from a previously reported study (Meule, Westenhöfer, \& Kübler, 2011) were reanalyzed to examine such interactive effects $(N=615,76 \%$ female $)$.

\section{RESULTS}

Higher rigid control was particularly associated with more frequent and intense food cravings, lower perceived self-regulatory success in weight regulation, and higher BMI at low levels of flexible control. A moderated serial multiple mediation model revealed that rigid control had an indirect effect on BMI through food cravings and perceived self-regulatory success, particularly when flexible control was low. These interactive effects could largely be replicated in a second study with female participants $(n=70)$.

\section{CONCLUSIONS}

The current findings replicate and extend previous reports in that high flexible control may "compensate" for high rigid control, that is, attenuate the effect of rigid control on eating behaviors and body weight. They also provide insights into the mediating mechanisms that link rigid and flexible control of eating behavior with BMI.

\section{KEY WORDS}

rigid control; flexible control; cognitive restraint; food cravings; body mass index

ORGANIZATION - University of Salzburg, Salzburg, Austria

AUthors' CONTRIBUtions - A: Study design - B: Data collection · C: Statistical analysis - D: Data interpretation .

E: Manuscript preparation · F: Literature search · G: Funds collection

Corresponding AUthor - Adrian Meule, Ph.D., University of Salzburg, Hellbrunner Straße 34, 5020 Salzburg, Austria, e-mail: adrian.meule@sbg.ac.at

TO CITE THIS ARTICLE - Meule, A. (2017). Interactive effects between flexible and rigid control of eating behavior

on body weight: a moderated serial multiple mediation model. Health Psychology Report, 5(4), 314-322.

doi: https://doi.org/10.5114/hpr.2017.70206

RECEIVED 06.06.2017 · REVIEWED 09.07.2017 · ACCEPTED 10.07.2017 • PUBLISHED 22.09.2017 


\section{BACKGROUND}

Restrained eating refers to the intention to restrict food intake deliberately in order to prevent weight gain or to promote weight loss (Tuschl, 1990). Westenhoefer (1991) proposed that the construct of cognitive restraint can be further divided into two dimensions: rigid and flexible control of eating behavior. Rigid control refers to an "all-or-nothing" approach to dieting such as eliminating "forbidden" foods from one's diet, skipping meals, or aiming for quick weight loss. Flexible control reflects a more balanced approach to dieting such as including a variety of foods in one's diet, eating small portions of food, or flexibly adjusting the amount of food eaten from meal to meal.

As both rigid and flexible control require the exertion of cognitive restraint, they are usually positively correlated with each other (Westenhoefer, 1991; Westenhoefer, Stunkard, \& Pudel, 1999). Similarly, both rigid and flexible control are associated with lower intuitive eating (Tylka, Calogero, \& Daníelsdóttir, 2015). However, when controlling for the influence of the other, they have dissociable or even opposing correlates. For example, while higher rigid control related to higher negative affect, lower body appreciation, poor interoceptive awareness, more binge eating, and higher food preoccupation, higher flexible control related to higher positive affect, higher body appreciation, better interoceptive awareness, less binge eating, and lower food preoccupation (Tylka et al., 2015). Similarly, higher rigid control has been found to correlate positively and higher flexible control to correlate negatively with disinhibited eating and body mass index (BMI; Meule, Westenhöfer, \& Kübler, 2011; Timko, Oelrich, \& Lowe, 2007; Timko \& Perone, 2005; Westenhoefer, Broeckmann, Munch, \& Pudel, 1994; Westenhoefer et al., 1999; Zyriax et al., 2012). Thus, it appears that flexible control is a more adaptive and functional dieting strategy with more favorable outcomes than rigid control.

In a previous study (Meule et al., 2011), rigid control related to more frequent and intense food cravings and lower perceived self-regulatory success in weight regulation. While flexible control related to higher perceived self-regulatory success, it was unrelated to food cravings. It was concluded from these results that food cravings mediated the relationship between rigid, but not flexible control and dieting success. However, it was not considered that rigid and flexible control may interact when predicting these outcomes (Westenhoefer et al., 1999). For example, Timko and Perone (2005) found that higher rigid control was more strongly related to higher BMI at low levels of flexible control than at high levels of flexible control. Thus, it appears that high levels of flexible control can "compensate" for high levels of rigid control, that is, exerting flexible control may reduce the influence of rigid control on higher BMI.

Another limitation of the analyses reported previously (Meule et al., 2011) is that they were restricted to two separate mediation models (i.e., (1) flexible control $\rightarrow$ food cravings $\rightarrow$ dieting success and (2) rigid control $\rightarrow$ food cravings $\rightarrow$ dieting success) without considering BMI. Contemporary mediation testing, however, allows one to combine interactive effects between variables (i.e., moderation) and mediation together in one model (i.e., moderated mediation) and to use more than one mediator in serial order (i.e., serial mediation; Hayes, 2015; Hayes \& Rockwood, in press). Based on the interactive effects between rigid and flexible control when predicting BMI (Timko \& Perone, 2005; Westenhoefer et al., 1999), it may be expected that rigid and flexible control will similarly interact when predicting food cravings and perceived self-regulatory success in weight regulation. Specifically, higher rigid control may particularly relate to more frequent and intense food cravings and lower perceived self-regulatory success when flexible control is low. As a result, flexible control may similarly moderate the indirect effect of rigid control on perceived self-regulatory success through food cravings such that this mediation effect may particularly be observed at low levels of flexible control.

Such a moderated mediation model can easily be extended by integrating BMI. Specifically, there is both cross-sectional (Meule \& Blechert, 2017) and longitudinal (Meule, Richard, \& Platte, 2017) support for a possible causal chain of more frequent and intense food cravings leading to lower perceived self-regulatory success in weight regulation, which in turn leads to higher BMI (i.e., food cravings $\rightarrow$ perceived self-regulatory success $\rightarrow$ BMI). Integrating these findings into a moderated serial multiple mediation model would suggest that rigid control may have an indirect effect on BMI through food cravings and perceived self-regulatory success in weight regulation (i.e., rigid control $\rightarrow$ food cravings $\rightarrow$ perceived self-regulatory success $\rightarrow$ BMI). These relationships, however, may be moderated by flexible control such that stronger relationships may be expected when flexible control is low (Figure 1).

Therefore, previously reported data (Meule et al., 2011) were reanalyzed by testing such a moderated serial multiple mediation model, which may provide a more comprehensive picture about relationships of rigid and flexible control of eating behavior with BMI and their mediating mechanisms (study 1). To further corroborate the resulting findings, data from a previously unpublished study in female students were analyzed (study 2). It was expected that there would be similar interactive effects between rigid and flexible control when predicting food cravings, perceived self-regulatory success, and BMI.
Flexible \& rigid control 


\section{STUDY 1}

\section{METHODS}

\section{Participants}

Data were used from a questionnaire-based study conducted online, which was approved by the institutional review board of the University of Würzburg. The recruitment procedure, a complete list of the measures used and other specifications are reported in detail elsewhere (Meule, Lutz, Vögele, \& Kübler, 2012; Meule et al., 2011). For the current analyses, data from 615 participants were available. Four-hundred and sixty-six participants (75.8\%) were women. Although the majority of participants were young adults with normal weight, there was a large range in age and BMI (Table 1).

\section{Questionnaires}

Rigid and flexible control of eating behavior. The German version of the rigid and flexible control scales (Westenhoefer et al., 1999) was used for the assessment of the two dimensions of cognitive restraint. The rigid control scale consists of 16 items (e.g., "I would rather skip a meal than stop eating in the middle of one., "Quick success is most important for me during a diet.") and the flexible control scale consists of 12 items (e.g., "I deliberately take small helpings as a means of weight control.", "If I eat a little bit more during one meal, I make up for it at the next meal."). They are scored with $0=$ false and $1=$ true. Thus, total scores can range between zero and 16 for the rigid control scale and between zero and 12 for the flexible control scale. Higher scores indicate higher rigid control and flexible control, respec-

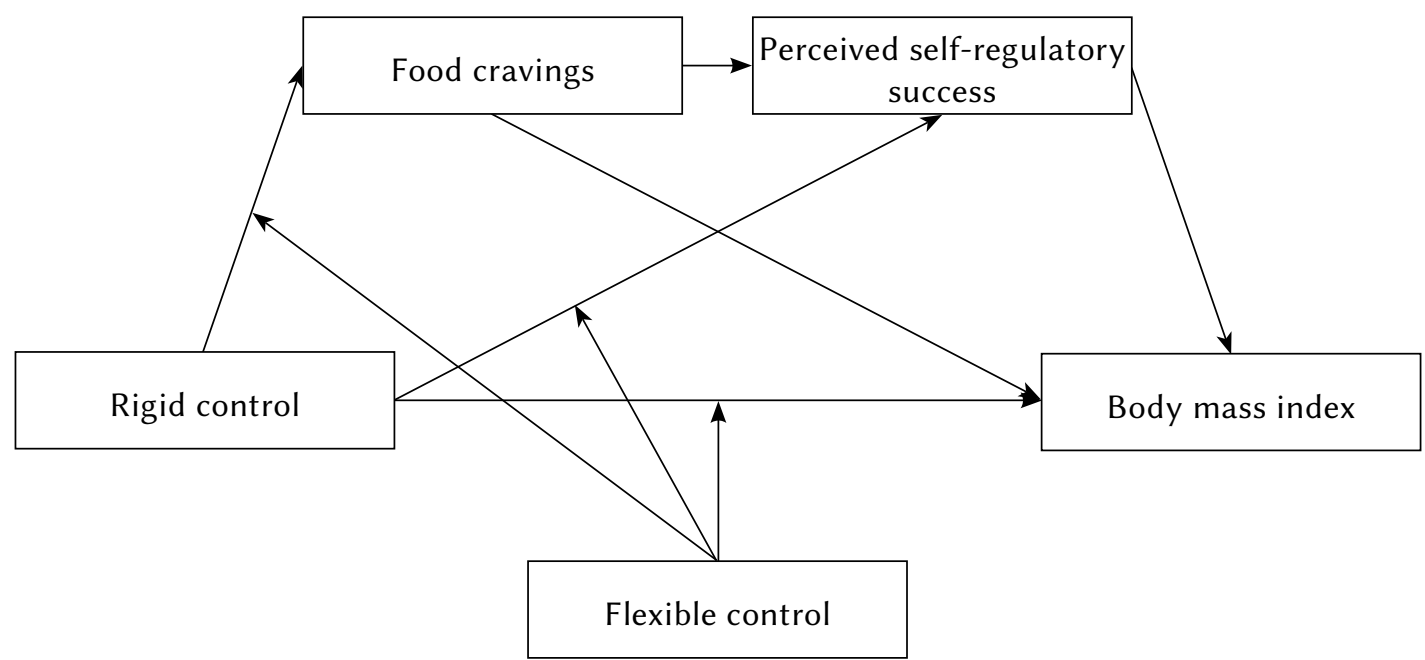

Figure 1. Conceptual moderated serial multiple mediation model. Scores on the Food Cravings Questionnaire-Trait and on the Perceived Self-Regulatory Success in Dieting Scale were used as mediators (in serial order) of the effect of rigid control of eating behavior on body mass index. Flexible control of eating behavior was used as a moderator of these indirect effects. Age and sex were included as covariates (not shown in this depiction).

Table 1

Descriptive statistics of and correlations between variables in study 1

\begin{tabular}{|c|c|c|c|c|c|c|c|c|c|c|}
\hline & $n$ & M & $S D$ & Range & 1. & 2. & 3. & 4. & 5. & 6. \\
\hline 1. Age (years) & 615 & 24.5 & 3.97 & $19-52$ & - & -.008 & -.064 & -.079 & .018 & $.110^{*}$ \\
\hline 2. Flexible control & 615 & 4.78 & 3.21 & $0-12$ & & - & $.590^{*}$ & .061 & $.162^{*}$ & -.057 \\
\hline 3. Rigid control & 615 & 4.19 & 3.43 & $0-16$ & & & - & $.438^{*}$ & $-.285^{*}$ & .220 \\
\hline $\begin{array}{l}\text { 4. Food Cravings } \\
\text { Questionnaire-Trait }\end{array}$ & 615 & 102 & 31.2 & $46-208$ & & & & - & $-.435^{*}$ & $.142^{*}$ \\
\hline $\begin{array}{l}\text { 5. Perceived Self-Reg- } \\
\text { ulatory Success in } \\
\text { Dieting Scale }\end{array}$ & 615 & 12.8 & 3.87 & $3-21$ & & & & & - & $-.411^{*}$ \\
\hline $\begin{array}{l}\text { 6. Body mass index } \\
\left(\mathrm{kg} / \mathrm{m}^{2}\right)\end{array}$ & 615 & 22.3 & 3.32 & $13.1-50.6$ & & & & & & - \\
\hline
\end{tabular}

Note. ${ }^{*} p<.050$ 
tively. Internal consistencies were $\alpha=.803$ (rigid control) and $\alpha=.822$ (flexible control).

\section{Food Cravings Questionnaire-Trait (FCQ-T)}

The German version of the FCQ-T (Cepeda-Benito, Gleaves, Williams, \& Erath, 2000; Meule, Lutz et al., 2012) was used for the assessment of the frequency and intensity of food cravings in general. The scale consists of 39 items (e.g., "If I am craving something, thoughts of eating it consume me., "If I give in to a food craving, all control is lost."), which are scored on a six-point scale ranging from $1=$ never $/$ not $a p$ plicable to $6=$ always. Thus, total scores can range between 39 and 234. Higher scores indicate more frequent and intense food craving experiences. Internal consistency was $\alpha=.961$.

\section{Perceived Self-Regulatory Success in Dieting Scale (PSRS)}

The German version of the PSRS (Fishbach, Friedman, \& Kruglanski, 2003; Meule, Papies, \& Kübler, 2012) was used for the assessment of perceived self-regulatory success in weight regulation. The scale consists of three items ("How successful are you in watching your weight?", "How successful are you in losing extra weight?", "How difficult do you find it to stay in shape?"), which are scored on a seven-point scale anchored 1 = successful/not difficult and 7 = very successful/ very difficult. Thus, total scores can range between 3 and 21. In the current study, participants were given an option to indicate when an item was not applicable to them. When participants chose this option, this was coded with 4 (i.e., the middle of the scale; cf. footnotes 1 and 2 in Meule, Papies et al., 2012). Higher scores indicate higher perceived self-regulatory success. Internal consistency was $\alpha=.704$.

\section{Data analyses}

Correlations between variables are displayed in Table 1. A moderated serial multiple mediation model was calculated with PROCESS for SPSS (Hayes, 2013). This model is based on three linear regression analyses (Hayes, 2015). In the first regression analysis, the first mediator (here: FCQ-T scores) is predicted by the independent variable (here: rigid control), the moderator (here: flexible control) and the interaction between the independent and the moderating variable (here: rigid $\times$ flexible control). In the second regression analysis, the second mediator (here: PSRS scores) is predicted by the independent variable, the moderator, their interaction, and the first mediator. In the third regression analysis, the outcome variable (here: BMI) is predicted by the independent variable, the moderator, their interaction, the first mediator, and the second mediator (cf. Table 2 and Figure 1).

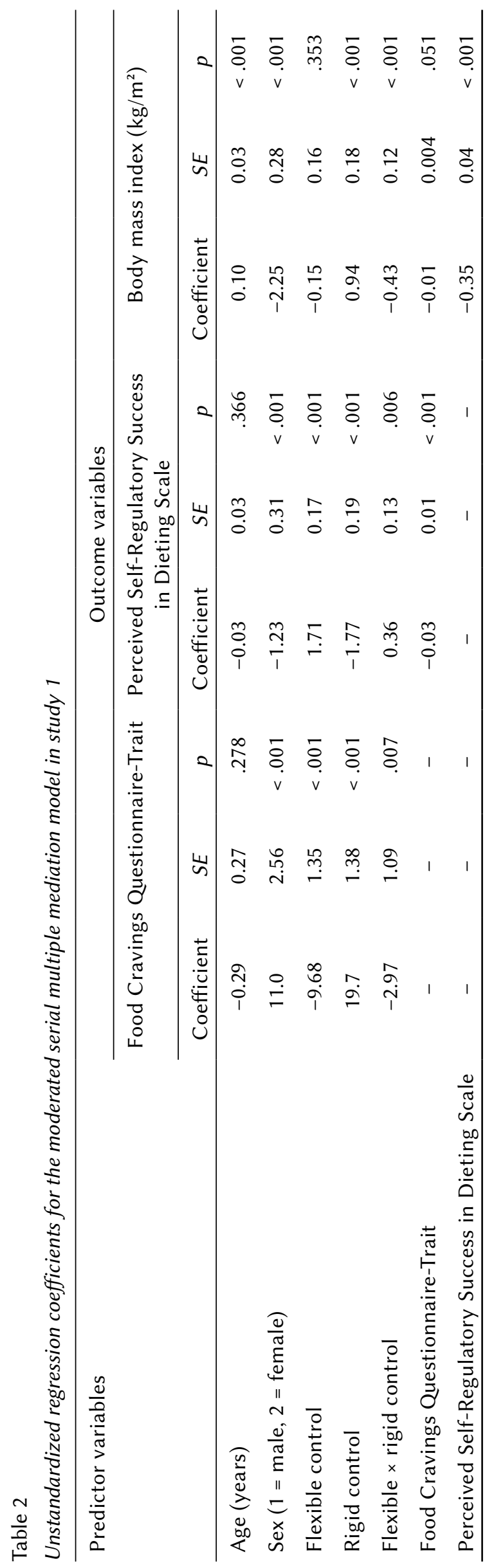

Flexible \& rigid control 
Age and sex were used as covariates. Rigid and flexible control scores were $z$-standardized before calculating the product term. Interactive effects between rigid and flexible control were examined with simple slopes analyses (Aiken \& West, 1991). Specifically, relationships between rigid control and the respective variables were examined at high $(+1 S D)$ and low $(-1 S D)$ values of flexible control (Figure 2). Indirect effects and the presence of moderated mediation were evaluated with $95 \%$ bias-corrected confidence intervals based on 10,000 bootstrap samples (Hayes, 2013, 2015).

\section{RESULTS}

Interactive effects between rigid and flexible control

Rigid and flexible control interactively predicted FCQ-T scores (Table 2). Probing the nature of this interaction revealed that - although higher rigid control was also related to more food cravings when flexible control was high $($ effect $=4.90, S E=0.44, p<.001)-$ the relationship between rigid control and food cravings was particularly strong when flexible control was low (effect $=6.63, S E=0.58, p<.001$, Figure 2 ).

Rigid and flexible control interactively predicted PSRS scores (Table 2). Probing the nature of this interaction revealed that - although higher rigid control was also related to lower success in weight regulation when flexible control was high (effect $=-0.56, S E=0.05, p<.001)-$ the relationship between rigid control and perceived self-regulatory success in weight regulation was particularly strong when flexible control was low (effect $=-0.82$, $S E=0.07, p<.001$, Figure 2).

Rigid and flexible control interactively predicted BMI (Table 2). Probing the nature of this interaction revealed that - although higher rigid control was also related to higher BMI when flexible control was high (effect $=0.30, S E=0.05, p<.001)-$ the relationship between rigid control and BMI was particularly strong when flexible control was low (effect $=0.63$, $S E=0.07, p<.001$, Figure 2).

\section{Indirect effects of rigid control on body mass index}

There was an indirect effect of rigid control on BMI through FCQ-T and PSRS scores (effect $=0.21$, $S E=0.05,95 \% \mathrm{CI}[0.12,0.30])$. Specifically, higher rigid control was indirectly related to higher BMI through more food cravings and lower perceived self-regulatory success. Flexible control, however, moderated this indirect effect of rigid control on BMI through FCQ-T and PSRS scores as indicated by the confidence interval for the index of moderated mediation not including zero $(95 \% \mathrm{CI}[-0.06,-0.01])$. Thus, the positive, indirect effect of rigid control on BMI was particularly pronounced at low levels of flexible control.

\section{STUDY 2}

\section{METHODS}

\section{Participants}

Data were used from an unpublished study, which was approved by the institutional review board of the University of Würzburg. Seventy women completed the rigid and flexible control scales, the FCQ-T, the PSRS, and other measures in the laboratory. Prior to completion of the questionnaires, participants' heart rate was recorded in resting condition. That is, no food-related experimental procedures were applied
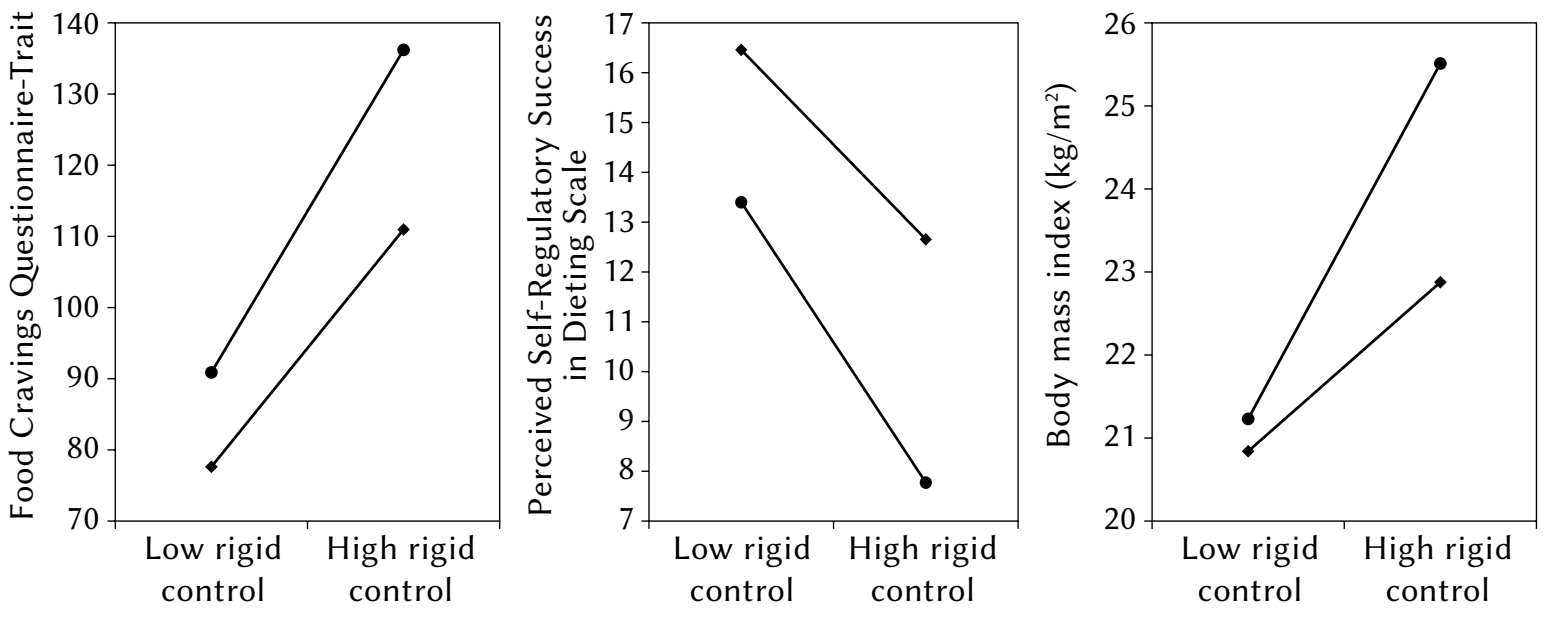

$\rightarrow$ Low flexible control

$\rightarrow$ High flexible control

Figure 2. Simple slopes probing the interaction between rigid and flexible control when predicting food cravings (left illustration), perceived self-regulatory success in weight regulation (middle illustration), and body mass index (right illustration) in study 1. 
Descriptive statistics of and correlations between variables in study 2

\begin{tabular}{|c|c|c|c|c|c|c|c|c|c|c|}
\hline & $n$ & $M$ & $S D$ & Range & 1. & 2. & 3. & 4. & 5. & 6. \\
\hline 1. Age (years) & 70 & 20.8 & 2.67 & $18-38$ & - & -.122 & -.138 & -.156 & .124 & .001 \\
\hline 2. Flexible control & 70 & 5.30 & 3.06 & $0-11$ & & - & $.597^{*}$ & .110 & .037 & -.026 \\
\hline 3. Rigid control & 70 & 4.06 & 3.39 & $0-13$ & & & - & .195 & $-.254^{*}$ & .139 \\
\hline $\begin{array}{l}\text { 4. Food Cravings } \\
\text { Questionnaire-Trait }\end{array}$ & 69 & 107 & 25.3 & $49-166$ & & & & - & $-.306^{*}$ & .232 \\
\hline $\begin{array}{l}\text { 5. Perceived Self-Reg- } \\
\text { ulatory Success in } \\
\text { Dieting Scale }\end{array}$ & 70 & 12.8 & 4.07 & $6-21$ & & & & & - & $-.557^{*}$ \\
\hline $\begin{array}{l}\text { 6. Body mass index } \\
\left(\mathrm{kg} / \mathrm{m}^{2}\right)\end{array}$ & 65 & 20.8 & 1.81 & $17.3-25.5$ & & & & & & - \\
\hline
\end{tabular}

Note. ${ }^{*} p<.050$.

that could have influenced questionnaire scores. Height and weight were measured by the experimenter after completion of the questionnaires. As outliers can have a larger impact on results in this smaller sample, data were screened for outliers using boxplots. Values that were outside of the "whiskers" were discarded from analyses (FCQ-T score of one participant and BMI of five participants), and thus sample size was slightly different for the different analyses (see Table 3). Similar to study 1, most participants were young adults with normal weight. In contrast to study 1 , however, range in age and BMI was more restricted (Table 3 ).

\section{QUESTIONNAIRES}

As in study 1, participants completed the German version of the rigid control scale $(\alpha=.799)$, flexible control scale $(\alpha=.789)$, FCQ-T $(\alpha=.936)$, and PSRS $(\alpha=.744)$.

\section{DATA ANALYSES}

Correlations between variables are displayed in Table 3. As sample size was not appropriate for testing such a complex model as in study 1 , interactive effects between rigid and flexible control on each variable were examined separately. Specifically, three moderation models were tested with PROCESS (Hayes, 2013) with rigid control, flexible control, and their interaction as predictor variables and FCQ-T scores, PSRS scores, and BMI as outcome variables (Table 4). Rigid and flexible control scores were $z$-standardized before calculating the product term. Interactive effects between rigid and flexible control were examined with simple slopes analyses (Aiken \& West, 1991). Specifically, relationships between rigid control and the respective variables were ex- amined at high $(+1 S D)$ and low $(-1 S D)$ values of flexible control (Figure 3).

\section{RESULTS}

Rigid and flexible control interactively predicted FCQ-T scores (Table 4). Probing the nature of this interaction revealed that rigid control was unrelated to food cravings when flexible control was high (effect = $0.10, S E=1.30, p=.938$ ), but higher rigid control was related to more food cravings when flexible control was low (effect $=4.31, S E=1.74, p=.016$, Figure 3).

Rigid control negatively and flexible control positively predicted PSRS scores, while the interaction was not significant (Table 4). Descriptively, however, the negative association between rigid control and perceived self-regulatory success was larger when flexible control was low (effect $=-0.64, S E=0.27$, $p=.022)$ than when flexible control was high (effect $=-0.45, S E=0.20, p=.026$, Figure 3 ).

The interaction between rigid and flexible control when predicting BMI was marginally significant (Table 4). Probing the nature of this interaction revealed that rigid control was unrelated to BMI when flexible control was high (effect $=0.04, S E=0.10, p=.682$ ), but higher rigid control was related to higher BMI when flexible control was low (effect $=0.30, S E=0.13$, $p=.022$, Figure 3).

\section{DISCUSSION}

The conclusion presented in a previous report (that food cravings mediate the relationship between rigid but not flexible control and dieting success; Meule et al., 2011) was incomplete. Instead, flexible control does indeed play a role in the proposed causal chain of rigid control leading to more food cravings and lower perceived self-regulatory success in weight
Flexible \& rigid control 
regulation. Specifically, study 1 showed that the effects of rigid control on food cravings, perceived self-regulatory success, and BMI were particularly strong when flexible control was low. These interactive effects were partially replicated in a smaller sample in study 2 . Moreover, the previously suggested mediation model could be extended in that there was an indirect effect of rigid control on BMI through food cravings and perceived self-regulatory success. This indirect effect was, again, particularly strong at low levels of flexible control.

The current findings suggest that, in the absence of flexible control, rigid control may particularly backfire and result in weight gain. Only a few studies have examined such moderating effects (Timko \& Perone, 2005; Westenhoefer et al., 1999), and, thus, the current results highlight the need to include interaction terms in studies investigating predictors of weight gain or treatment success in weight-loss interventions. The results also suggest possible mediating mechanisms that link eating control strategies and body weight. While the current analyses were based on cross-sectional data, the direction of effects (e.g., food cravings $\rightarrow$ perceived self-regulatory success $\rightarrow$ BMI) is in line with similar mediation models based on cross-sectional (Meule $\&$ Blechert, 2017) and longitudinal data (Meule et al., 2017). Therefore, it may well be that exhibiting high rigid control of eating behavior with concomitant low flexible control may be causally involved in increased food craving experiences and giving into them, which in turn lead to decreased self-regulatory success and weight gain.

Interpretation of the findings is limited by the sample characteristics as participants were primarily young females with normal weight. Although controlling for age and sex in study 1 did not influence the results, future studies need to replicate the findings in other samples such as men, older adults, or individuals with obesity. Furthermore, interventional studies that record eating behavior (e.g., occurrence of food cravings) in daily life (e.g., with ecological momentary assessment) are needed in order to provide support for ecological validity of the current findings as well as for the assumed causal direction of rigid $\times$ flexible control leading to more food cravings.

In conclusion, the current studies provide a model that recognizes under which circumstances rigid control of eating behavior may particularly lead to higher body weight (i.e., when flexible control is absent). In other words, high flexible control appears to attenuate the effect of rigid control on body weight. Additionally, this model also suggests possible mechanisms that may explain how rigid control translates into higher body weight (i.e., through increased food cravings and decreased self-regulatory success in weight regulation). 

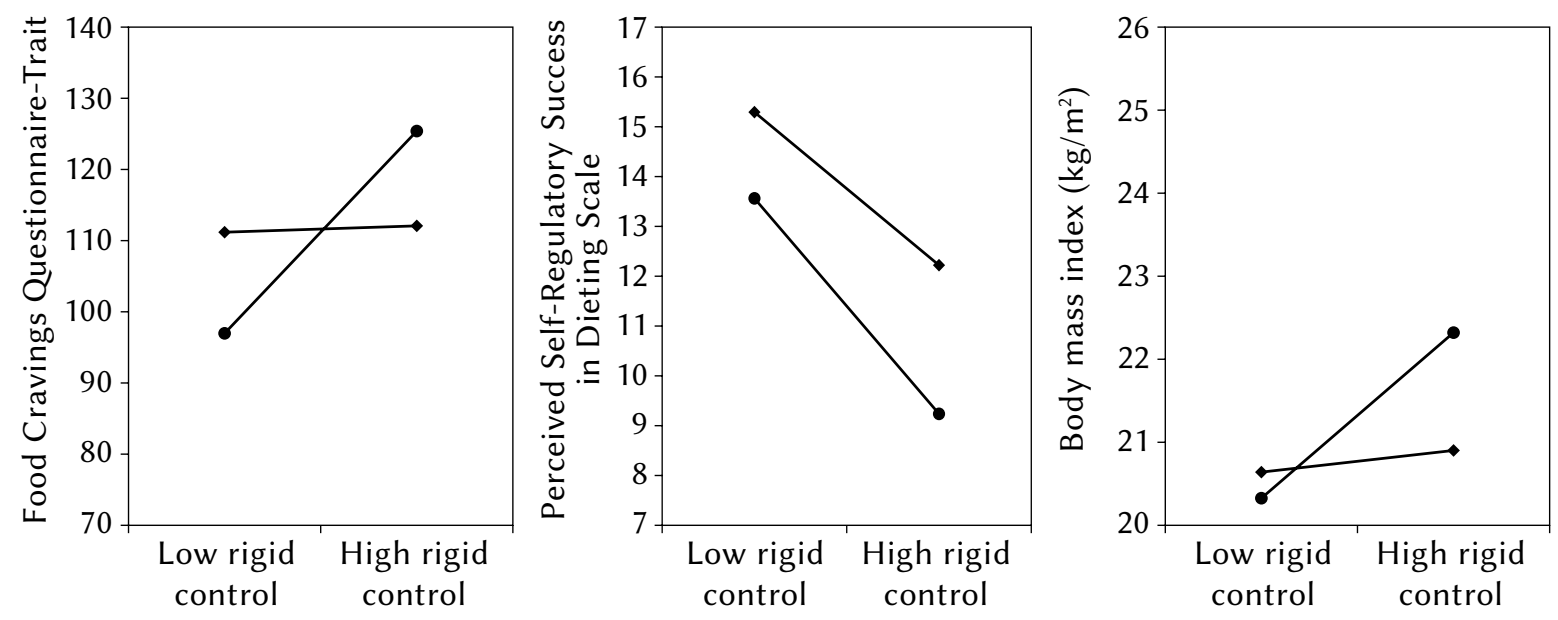

Flexible \& rigid control

Figure 3. Simple slopes probing the interaction between rigid and flexible control when predicting food cravings (left illustration), perceived self-regulatory success in weight regulation (middle illustration), and body mass index (right illustration) in study 2.

\section{ACKNOWLEDGMENT}

The author would like to thank Sabine Meininger for collecting data for study 2 .

\section{REFERENCES}

Aiken, L. S., \& West, S. G. (1991). Multiple regression: Testing and interpreting interactions. Thousand Oaks, CA: Sage.

Cepeda-Benito, A., Gleaves, D. H., Williams, T. L., \& Erath, S. A. (2000). The development and validation of the state and trait Food-Cravings Questionnaires. Behavior Therapy, 31, 151-173.

Fishbach, A., Friedman, R. S., \& Kruglanski, A. W. (2003). Leading us not unto temptation: Momentary allurements elicit overriding goal activation. Journal of Personality and Social Psychology, 84, 296-309.

Hayes, A. F. (2013). Introduction to Mediation, Moderation, and Conditional Process Analysis. New York: The Guilford Press.

Hayes, A. F. (2015). An index and test of linear moderated mediation. Multivariate Behavioral Research, 50, 1-22.

Hayes, A. F., \& Rockwood, N. J. (in press). Regression-based statistical mediation and moderation analysis in clinical research: Observations, recommendations, and implementation. Behaviour Research and Therapy.

Meule, A., \& Blechert, J. (2017). Indirect effects of trait impulsivity on body mass. Eating Behaviors, 26, 66-69.

Meule, A., Lutz, A., Vögele, C., \& Kübler, A. (2012). Food cravings discriminate differentially between successful and unsuccessful dieters and non-dieters. Validation of the Food Cravings Questionnaires in German. Appetite, 58, 88-97.
Meule, A., Papies, E. K., \& Kübler, A. (2012). Differentiating between successful and unsuccessful dieters: Validity and reliability of the Perceived Self-Regulatory Success in Dieting Scale. Appetite, 58, 822-826.

Meule, A., Richard, A., \& Platte, P. (2017). Food cravings prospectively predict decreases in perceived self-regulatory success in dieting. Eating Behaviors, 24, 34-38.

Meule, A., Westenhöfer, J., \& Kübler, A. (2011). Food cravings mediate the relationship between rigid, but not flexible control of eating behavior and dieting success. Appetite, 57, 582-584.

Timko, C. A., Oelrich, C., \& Lowe, M. R. (2007). Prospective relationship of rigid and flexible control and weight loss. Verhaltenstherapie, 17, 84-89.

Timko, C. A., \& Perone, J. (2005). Rigid and flexible control of eating behavior in a college population. Eating Behaviors, 6, 119-125.

Tuschl, R. J. (1990). From dietary restraint to binge eating: some theoretical considerations. Appetite, 14, 105-109.

Tylka, T. L., Calogero, R. M., \& Daníelsdóttir, S. (2015). Is intuitive eating the same as flexible dietary control? Their links to each other and well-being could provide an answer. Appetite, 95, 166-175.

Westenhoefer, J. (1991). Dietary restraint and disinhibition: Is restraint a homogeneous construct? Appetite, 16, 45-55.

Westenhoefer, J., Broeckmann, P., Munch, A. K., \& Pudel, V. (1994). Cognitive control of eating behaviour and the disinhibition effect. Appetite, 23, 27-41.

Westenhoefer, J., Stunkard, A. J., \& Pudel, V. (1999). Validation of the flexible and rigid control dimensions of dietary restraint. International Journal of Eating Disorders, 26, 53-64. 
Zyriax, B. C., Wolf, C., Schlüter, A., Khattak, A. H., Westenhoefer, J., \& Windler, E. (2012). Association of cognitive dietary restraint and disinhibition with prediabetes-cross-sectional and longitudinal data of a feasibility study in German employees. Public Health Nutrition, 15, 860-867.

Adrian Meule 